\title{
On the Difference Sequence Space $\ell_{p}\left(\hat{T}^{q}\right)$
}

\author{
Pınar Zengin Alp* and Merve İlkhan
}

\begin{abstract}
In this study, we introduce a new matrix $\hat{T}^{q}=\left(\hat{t}_{n k}^{q}\right)$ by

$$
\hat{t}_{n k}^{q}=\left\{\begin{array}{ccc}
\frac{q_{n}}{Q_{n}} t_{n} & , \quad k=n \\
\frac{q_{k}}{Q_{n}} t_{k}-\frac{q_{k+1}}{Q_{n}} \frac{1}{t_{k+1}} & , \quad k<n \\
0 & , \quad k>n .
\end{array}\right.
$$

where $t_{k}>0$ for all $n \in \mathbb{N}$ and $\left(t_{n}\right) \in c \backslash c_{0}$. By using the matrix $\hat{T}^{q}$, we introduce the sequence space $\ell_{p}\left(\hat{T}^{q}\right)$ for $1 \leq p \leq \infty$. In addition, we give some theorems on inclusion relations associated with $\ell_{p}\left(\hat{T}^{q}\right)$ and find the $\alpha-, \beta-, \gamma$ - duals of this space. Lastly, we analyze the necessary and sufficient conditions for an infinite matrix to be in the classes $\left(\ell_{p}\left(\hat{T}^{q}\right), \lambda\right)$ or $\left(\lambda, \ell_{p}\left(\hat{T}^{q}\right)\right)$, where $\lambda \in\left\{\ell_{1}, c_{0}, c, \ell_{\infty}\right\}$.

Keywords: sequence spaces; matrix transformations; Schauder basis; $\alpha-, \beta-, \gamma-d$ uals.

AMS Subject Classification (2010): Primary: 11B39; Secondary: 46A45; $46 B 45$.

*Corresponding author
\end{abstract}

\section{Introduction and preliminaries}

Let $\omega$ denote the set of all real or complex sequences and $\lambda$ and $\mu$ be subsets of $\omega$. We shall use $\sup _{k}$ instead of $\sup _{k \in \mathbb{N}}$ and $\sum_{k}$ instead of $\sum_{k=0}^{\infty}$, where $\mathbb{N}=\{0,1,2, \ldots\}$ to provide convenience. Also, if $u=\left(u_{k}\right)_{k=0}^{\infty} \in \omega$, we simply denote it by $u=\left(u_{k}\right)$. Further, $e=(1,1, \ldots)$ and $e^{(k)}$ is the sequence whose $k$ th term is 1 and the other terms are 0 , that is, $e^{(k)}=\left(e_{0}^{(k)}, e_{1}^{(k)}, \ldots, e_{k}^{(k)}, \ldots\right)=(0,0, \ldots, 1, \ldots)$. Any vector subspace of $\omega$ is called a sequence space. By $\ell_{\infty}, c, c_{0}$ and $\ell_{p}(1 \leq p<\infty)$, we denote the spaces of all bounded, convergent, null sequences and $p$-absolutely convergent series, respectively.

$\lambda$ with a linear topology is called a $K$-space provided each of the maps $p_{n}: \lambda \rightarrow \mathbb{C}$ defined by $p_{n}(x)=x_{n}$ is continuous for all $n \in \mathbb{N}$, where $\mathbb{C}$ is the set of all complex numbers. If a $K$-space $\lambda$ is a complete metric space, it is said to be an $F K$-space. A normed $F K$-space is defined as a $B K$-space, hence, a $B K$-space is a Banach sequence space. For instance, the sequence space $\ell_{\infty}$ is a $B K$-space with the norm given by $\|u\|_{\ell_{\infty}}=\sup _{k}\left|u_{k}\right|$. Further, $\ell_{p}$ is a complete $p$-normed space with respect to the usual $p$-norm defined by

$$
\|u\|_{\ell_{p}}=\sum_{k}\left|u_{k}\right|^{p} \quad(0<p<1)
$$

and $\ell_{p}$ is a $B K$-space with respect to $\ell_{p}$-norm defined by

$$
\|u\|_{\ell_{p}}=\left(\sum_{k}\left|u_{k}\right|^{p}\right)^{1 / p} \quad(1 \leq p<\infty) .
$$

Let $B=\left(b_{n k}\right)$ be an infinite matrix of real or complex numbers $b_{n k}$, where $n, k \in \mathbb{N}$. Then $B$ defines a matrix mapping from $\lambda$ into $\mu$ and we write $B: \lambda \rightarrow \mu$ if for every sequence $u=\left(u_{k}\right) \in \lambda$, the sequence $B u=\left(B_{n}(u)\right)$, the

Received : 28-07-2019, Accepted : 01-09-2019 
$B$-transform of $u$, is in $\mu$, where

$$
B_{n}(u)=\sum_{k} b_{n k} u_{k} \quad(n \in \mathbb{N}) .
$$

By $(\lambda, \mu)$, we denote the class of all infinite matrices that map $\lambda$ into $\mu$. Hence $A \in(\lambda, \mu)$ if and only if the series $\sum_{k} b_{n k} u_{k}$ converges for each $n \in \mathbb{N}$ and every $u \in \lambda$, and $B u \in \mu$ for all $u \in \lambda$. If $\lambda$ and $\mu$ are two arbitrary Banach spaces, then $\mathcal{B}(\lambda, \mu)$ denotes the set of all bounded linear operators from $\lambda$ into $\mu$.

The matrix domain $\lambda_{B}$ of an infinite matrix $B$ is defined by

$$
\lambda_{B}=\left\{u=\left(u_{k}\right) \in \omega: B u \in \lambda\right\}
$$

which is also a sequence space.

In the literature, there are many papers related to new sequence spaces constructed by means of the matrix domain of a special triangle. See, for example [1]-[20]. For more information about matrix domains of triangles, one can see [21].

A sequence $\left(\beta_{n}\right)$ in normed space $\lambda$ is called a Schauder basis for $\lambda$ if for every $u \in \lambda$ there is a unique sequence $\left(\alpha_{n}\right)$ of scalars such that $u=\sum_{n} \alpha_{n} \beta_{n}$, i.e.,

$$
\lim _{m \rightarrow \infty}\left\|u-\sum_{n=0}^{m} \alpha_{n} \beta_{n}\right\|=0 .
$$

By $c s_{0}, c s$ and $b s$, we denote the set of all convergent to zero, convergent and bounded series, respectively, that is, $c s_{0}=\left\{u=\left(u_{k}\right) \in \omega:\left(\sum_{k=0}^{n} u_{k}\right)_{n=0}^{\infty} \in c_{0}\right\}, c s=\left\{u=\left(u_{k}\right) \in \omega:\left(\sum_{k=0}^{n} u_{k}\right)_{n=0}^{\infty} \in c\right\}$ and $b s=\left\{u=\left(u_{k}\right) \in \omega:\right.$ $\left.\left(\sum_{k=0}^{n} u_{k}\right)_{n=0}^{\infty} \in \ell_{\infty}\right\}$, and we define the norm on $c s_{0}, c s$ and $b s$ by $\|u\|_{c s_{0}}=\|u\|_{c s}=\|u\|_{b s}=\sup _{n}\left|\sum_{k=0}^{n} u_{k}\right|$. For all $z \in \omega$, we write $z^{-1} * \mu=\left\{x \in \omega: x z=\left(x_{k} z_{k}\right) \in \mu\right\}$. The set $Z=M(\lambda, \mu)=\cap_{u \in \lambda} u^{-1} * \mu=\{a \in \omega: a u \in$ $\mu$ for all $u \in \lambda\}$ is called the multiplier space of $\lambda$ and $\mu$. In the special case, where $\mu=\ell_{1}, \mu=c s$ or $\mu=b s$, the multiplier spaces $\lambda^{\alpha}=M\left(\lambda, \ell_{1}\right), \lambda^{\beta}=M(\lambda, c s)$ and $\lambda^{\gamma}=M(\lambda, b s)$ are called the $\alpha-, \beta$ - and $\gamma$-duals of $\lambda$.

Throughout this paper, we assume that $p, q \geq 1$ with $\frac{1}{p}+\frac{1}{q}=1$ and denote the collection of all finite subsets of $\mathbb{N}$ by $\mathcal{F}$.

The difference operator $\Delta: \omega \rightarrow \omega$ is defined by $\Delta u=\left(\Delta u_{k}\right)=\left(u_{k}-u_{k-1}\right)$ or $\Delta u=\left(\Delta u_{k}\right)=\left(u_{k-1}-u_{k}\right)$ for all $u=\left(u_{k}\right) \in \omega$. When $\lambda$ is a sequence space, the matrix domain $\lambda_{\Delta}$ is called the difference sequence space. For the first time, Kizmaz [22] gave the notion of difference sequence spaces as

$$
\lambda(\Delta)=\left\{u=\left(u_{k}\right) \in \omega:\left(u_{k}-u_{k-1}\right) \in \lambda\right\}
$$

for $\lambda=\ell_{\infty}, c$ and $c_{0}$. After Kızmaz, Et and Çolak [23] defined the generalized difference sequence spaces

$$
\begin{gathered}
\ell_{\infty}\left(\Delta^{m}\right)=\left\{u=\left(u_{k}\right) \in \omega: \Delta^{m} u \in \ell_{\infty}\right\}, \\
c\left(\Delta^{m}\right)=\left\{u=\left(u_{k}\right) \in \omega: \Delta^{m} u \in c\right\}
\end{gathered}
$$

and

$$
c_{0}\left(\Delta^{m}\right)=\left\{u=\left(u_{k}\right) \in \omega: \Delta^{m} u \in c_{0}\right\},
$$

where $m \in \mathbb{N}, \Delta^{m} u=\left(\Delta^{m} u_{k}\right)=\left(\Delta^{m-1} u_{k}-\Delta^{m-1} u_{k+1}\right)$ and so that

$$
\Delta^{m} u_{k}=\sum_{i=0}^{m}(-1)^{i}\left(\begin{array}{c}
m \\
i
\end{array}\right) u_{k+i} .
$$

The difference space

$$
b v_{p}=\left\{u=\left(u_{k}\right) \in \omega:\left(u_{k}-u_{k-1}\right) \in \ell_{p}\right\} \quad(0<p<\infty)
$$

was studied by Altay and Başar [24] for $0<p<1$ and in the case $1 \leq p \leq \infty$ Başar and Altay [25], and Çolak et al [26]. Recently, for $\lambda \in\left\{\ell_{p}, c_{0}, c, \ell_{\infty}\right\}(1 \leq p<\infty)$, Kirişçi and Başar [4] introduced the generalized difference sequence space

$$
\widehat{\lambda}=\left\{u=\left(u_{k}\right): \in \omega: B(r, s) u=\left((B(r, s) u)_{k}\right) \in \lambda\right\},
$$


where $B(r, s) u$ is the sequence defined by $(B(r, s) u)_{k}=r u_{k}+s u_{k-1}$ for all $k \in \mathbb{N}$ and $r, s \in \mathbb{R} \backslash\{0\}$.

In [27], the Fibonacci band matrix $\widehat{F}$ is defined by using Fibonacci numbers. Also, in [27] the Fibonacci difference sequence spaces $\ell_{p}(\widehat{F})$ and $\ell_{\infty}(\widehat{F})$ are introduced.

The Riesz matrix $R_{q}=\left(r_{n k}\right)$ is defined by

$$
r_{n k}=\left\{\begin{array}{cll}
\frac{q_{k}}{Q_{n}} & , \quad 0 \leq k \leq n \\
0 & , \quad k>n
\end{array}\right.
$$

for all $k, n \in \mathbb{N}$ and where $\left(q_{k}\right)$ is the sequence of positive numbers and $Q_{n}=\sum_{k=0}^{n} q_{k}$ for all $n \in \mathbb{N}$. In [28], the paranormed Riesz sequence space is introduced.

In [29], the band matrix $T=\left(t_{n k}\right)$ is defined by

$$
t_{n k}=\left\{\begin{array}{ccc}
t_{n} & , \quad k=n \\
-\frac{1}{t_{n}} & , \quad k<n \\
0 & , \quad k>n
\end{array}\right.
$$

where $t_{n}>0$ for all $n \in \mathbb{N}$ and $t=\left(t_{n}\right) \in c \backslash c_{0}$. Also in [29] the difference sequence spaces are introduced as follows:

$$
\ell_{p}(T)=\left\{u=\left(u_{n}\right) \in \omega: \sum_{n}\left|t_{n} u_{n}-\frac{1}{t_{n}} u_{n-1}\right|^{p}<\infty\right\}(1 \leq p<\infty)
$$

and

$$
\ell_{\infty}(T)=\left\{u=\left(u_{n}\right) \in \omega: \sup _{n}\left|t_{n} u_{n}-\frac{1}{t_{n}} u_{n-1}\right|<\infty\right\} .
$$

For more information on some new difference sequence spaces we refer to [30]-[37].

The paper is organized so that this section is followed by three sections. In Section 2 we give the definition of a new matrix and introduce the sequence spaces $\ell_{p}\left(\hat{T}^{q}\right)$ and $\ell_{\infty}\left(\hat{T}^{q}\right)$, where $1 \leq p<\infty$. We prove that $\ell_{p}\left(\hat{T}^{q}\right)$ and $\ell_{\infty}\left(\hat{T}^{q}\right)$ are Banach spaces with respect to the norm defined on these spaces. Further, we establish some inclusion theorems related to the space $\ell_{p}\left(\hat{T}^{q}\right)$, where $1 \leq p \leq \infty$. In section 3 we determine the $\alpha-, \beta-, \gamma$ - duals of the space $\ell_{p}\left(\hat{T}^{q}\right)$ for $1 \leq p \leq \infty$. In the last section we characterize the classes $\left(\ell_{p}\left(\hat{T}^{q}\right), \lambda\right)$ and $\left(\lambda, \ell_{p}\left(\hat{T}^{q}\right)\right)$ for $\lambda \in\left\{\ell_{1}, c_{0}, c, \ell_{\infty}\right\}$.

\section{The difference sequence space $\ell_{p}\left(\hat{T}^{q}\right)$}

In this section, we introduce a new matrix $\hat{T}^{q}$ by multiplying Riesz matrix and the band matrix $T$ and introduce the difference sequence space $\ell_{p}\left(\hat{T}^{q}\right)$ derived by using this matrix, where $1 \leq p \leq \infty$. Also, we give some theorems which give inclusion relations corcerning this space. By multiplying these matrices we derive a new matrix $\hat{T}^{q}=\left(\hat{t}_{n k}^{q}\right)$ as

$$
\hat{t}_{n k}^{q}=\left\{\begin{array}{cc}
\frac{q_{n}}{Q_{n}} t_{n} & , \quad k=n \\
\frac{q_{k}}{Q_{n}} t_{k}-\frac{q_{k+1}}{Q_{n}} \frac{1}{t_{k+1}} & , \quad k<n \\
0 & , \quad k>n .
\end{array}\right.
$$

$\left(\hat{T}^{q}\right)^{-1}=\left(\left(\hat{t}^{q}\right)_{n k}^{-1}\right)$, the inverse of $\hat{T}^{q}$ can be easily computed as

$$
\left(\hat{t}^{q}\right)_{n k}^{-1}=\left\{\begin{array}{cl}
Q_{k}\left[\frac{1}{q_{k}}\left(t_{k} \prod_{j=k}^{n} \frac{1}{t_{j}^{2}}\right)^{\frac{Q_{n}}{q_{n}} \frac{1}{t_{n}}}-\frac{1}{q_{k+1}}\left(t_{k+1} \prod_{j=k+1}^{n} \frac{1}{t_{j}^{2}}\right)\right], & k=n \\
0 & , \quad k>n .
\end{array}\right.
$$

Now, let give the definitions of the difference sequence spaces $\ell_{p}\left(\hat{T}^{q}\right)$ and $\ell_{\infty}\left(\hat{T}^{q}\right)$ derived by this matrix

$$
\ell_{p}\left(\hat{T}^{q}\right)=\left\{u=\left(u_{n}\right) \in \omega: \sum_{n}\left|\frac{1}{Q_{n}} \sum_{k=0}^{n} q_{k}\left(t_{k} u_{k}-\frac{u_{k-1}}{t_{k}}\right)\right|^{p}<\infty\right\} \quad(1 \leq p<\infty)
$$


and

$$
\ell_{\infty}\left(\hat{T}^{q}\right)=\left\{u=\left(u_{n}\right) \in \omega: \sup _{n}\left|\frac{1}{Q_{n}} \sum_{k=0}^{n} q_{k}\left(t_{k} u_{k}-\frac{u_{k-1}}{t_{k}}\right)\right|<\infty\right\} .
$$

For the $\hat{T}^{q}$-transform of a sequence $u=\left(u_{n}\right)$, we will use the sequence $\hat{u}=\left(\hat{u}_{n}\right)$ defined as

$$
\hat{u}_{n}=\hat{T}_{n}^{q}(u)=\frac{1}{Q_{n}} \sum_{k=0}^{n} q_{k}\left(t_{k} u_{k}-\frac{u_{k-1}}{t_{k}}\right)(n \in \mathbb{N}) .
$$

Theorem 2.1. For $1 \leq p \leq \infty, \ell_{p}\left(\hat{T}^{q}\right)$ is a Banach space with the norm $\|u\|_{\ell_{p}\left(\hat{T}^{q}\right)}=\left\|\hat{T}^{q} u\right\|_{\ell_{p}}$, defined as,

$$
\|u\|_{\ell_{p}\left(\hat{T}^{q}\right)}=\left\{\begin{array}{cl}
\left(\sum_{n}\left|\hat{T}_{n}^{q}(u)\right|^{p}\right)^{1 / p} & , \quad 1 \leq p<\infty \\
\sup _{n}\left|\hat{T}_{n}^{q}(u)\right| & , \quad p=\infty
\end{array}\right.
$$

Proof. If we assume that $\|u\|_{\ell_{p}\left(\hat{T}^{q}\right)}=0$. Then, $\left\|\hat{T}^{q} u\right\|_{\ell_{p}}=0$ and since $\|\cdot\|_{\ell_{p}}$ is a norm we have $\hat{T}^{q} u=\theta$. Since it is known that $\hat{T}^{q}$ is invertible, we have $u=\theta$.

Let $\alpha \in \mathbb{C}$ and $u \in \ell_{p}\left(\hat{T}^{q}\right)$. Then,

$$
\begin{aligned}
\|\alpha u\|_{\ell_{p}\left(\hat{T}^{q}\right)} & =\left\|\hat{T}^{q}(\alpha u)\right\|_{\ell_{p}}=\left\|\alpha \hat{T}^{q} u\right\|_{\ell_{p}} \\
& =|\alpha|\left\|\hat{T}^{q} u\right\|_{\ell_{p}}=|\alpha|\|u\|_{\ell_{p}\left(\hat{T}^{q}\right)} .
\end{aligned}
$$

Finally, for $u, v \in \ell_{p}\left(\hat{T}^{q}\right)$ we have

$$
\begin{aligned}
\|u+v\|_{\ell_{p}\left(\hat{T}^{q}\right)} & =\left\|\hat{T}^{q}(u+v)\right\|_{\ell_{p}}=\left\|\hat{T}^{q} u+\hat{T}^{q} v\right\|_{\ell_{p}} \\
& \leq\left\|\hat{T}^{q} u\right\|_{\ell_{p}}+\left\|\hat{T}^{q} v\right\|_{\ell_{p}}=\|u\|_{\ell_{p}\left(\hat{T}^{q}\right)}+\|v\|_{\ell_{p}\left(\hat{T}^{q}\right)}
\end{aligned}
$$

and so the triangle inequality holds.

This means that, $\left(\ell_{p}\left(\hat{T}^{q}\right),\|\cdot\|_{\ell_{p}\left(\hat{T}^{q}\right)}\right)$ is a normed sequence space for $1 \leq p \leq \infty$. To show that $\ell_{p}\left(\hat{T}^{q}\right)$ is a Banach space, let $\left(u_{n}\right)$ be a Cauchy sequece in $\ell_{p}\left(\hat{T}^{q}\right)$. Then, $\left(\hat{u}_{n}\right)$ is a sequence in $\ell_{p}$. Obviously,

$$
\begin{aligned}
\left\|u_{n}-u_{m}\right\|_{\ell_{p}\left(\hat{T}^{q}\right)} & =\left\|\hat{T}^{q}\left(u_{n}-u_{m}\right)\right\|_{\ell_{p}} \\
& =\left\|\hat{T}^{q} u_{n}-\hat{T}^{q} u_{m}\right\|_{\ell_{p}}=\left\|\hat{u}_{n}-\hat{u}_{m}\right\|_{\ell_{p}},
\end{aligned}
$$

hence, $\left(\hat{u}_{n}\right)$ is a Cauchy sequence in $\ell_{p}$. Since $\left(\ell_{p},\|\cdot\|_{\ell_{p}}\right)$ is a Banach space, there exists $\hat{u} \in \ell_{p}$ such that $\lim _{n \rightarrow \infty} \hat{u}_{n}=\hat{u}$ in $\ell_{p}$. Since $u=\left(\hat{T}^{q}\right)^{-1} \hat{u}$, we have

$$
\begin{aligned}
\lim _{n \rightarrow \infty}\left\|u_{n}-u\right\|_{\ell_{p}\left(\hat{T}^{q}\right)} & =\lim _{n \rightarrow \infty}\left\|\hat{T}^{q}\left(u_{n}-u\right)\right\|_{\ell_{p}} \\
& =\lim _{n \rightarrow \infty}\left\|\hat{T}^{q} u_{n}-\hat{T}^{q} u\right\|_{\ell_{p}}=\lim _{n \rightarrow \infty}\left\|\hat{u}_{n}-\hat{u}\right\|_{\ell_{p}}=0 .
\end{aligned}
$$

Hence $\lim _{n \rightarrow \infty} u_{n}=u$ in $\ell_{p}\left(\hat{T}^{q}\right)$, where $u \in \ell_{p}\left(\hat{T}^{q}\right)$.

Remark 2.1. $\ell_{p}\left(\hat{T}^{q}\right)$ is a $B K$-space for $1 \leq p \leq \infty$.

Theorem 2.2. The sequence spaces $\ell_{p}\left(\hat{T}^{q}\right)$ and $\ell_{p}$ are linearly isomorphic; that is, $\ell_{p}\left(\hat{T}^{q}\right) \cong \ell_{p}$ for $1 \leq p \leq \infty$.

Proof. It must be shown that there exists a linear bijection between the spaces $\ell_{p}\left(\hat{T}^{q}\right)$ and $\ell_{p}$ for $1 \leq p \leq \infty$. Let $\hat{T}^{q}$ be the transformation defined from $\ell_{p}\left(\hat{T}^{q}\right)$ to $\ell_{p}$ by $u \rightarrow \hat{u}=\hat{T}^{q} u=\left(\hat{T}_{n}^{q}(u)\right)$. Then, we have $\hat{T}^{q} u=\hat{u} \in \ell_{p}$ for every $u \in \ell_{p}\left(\hat{T}^{q}\right)$. Hence, $\hat{T}^{q}$ is a linear transformation. Also, $\hat{T}^{q}$ is injective since $u=\theta$ whenever $\hat{T}^{q} u=\theta$.

Moreover, let $v=\left(v_{n}\right) \in \ell_{p}$ be given for $1 \leq p \leq \infty$ and define the sequence $u=\left(u_{n}\right)$ as follows:

$$
u_{n}=\sum_{k=0}^{n} Q_{k}\left[\frac{1}{q_{k}}\left(t_{k} \prod_{j=k}^{n} \frac{1}{t_{j}^{2}}\right)-\frac{1}{q_{k+1}}\left(t_{k+1} \prod_{j=k+1}^{n} \frac{1}{t_{j}^{2}}\right)\right] v_{k} \quad(n \in \mathbb{N}) .
$$


Then, by combining (2.1) and (2.2), we get for every $n \in \mathbb{N}$

$$
\begin{aligned}
\hat{T}_{n}^{q}(u) & =\frac{1}{Q_{n}} \sum_{k=0}^{n} q_{k}\left(t_{k} \sum_{r=0}^{k} Q_{r}\left[\frac{1}{q_{r}}\left(t_{r} \prod_{j=r}^{k} \frac{1}{t_{j}^{2}}\right)-\frac{1}{q_{r+1}}\left(t_{r+1} \prod_{j=r+1}^{k} \frac{1}{t_{j}^{2}}\right)\right] v_{r}\right) \\
& -\frac{1}{Q_{n}} \sum_{k=0}^{n} q_{k}\left(\frac{1}{t_{k}} \sum_{r=0}^{k-1} Q_{r}\left[\frac{1}{q_{r}}\left(t_{r} \prod_{j=r}^{k-1} \frac{1}{t_{j}^{2}}\right)-\frac{1}{q_{r+1}}\left(t_{r+1} \prod_{j=r+1}^{k-1} \frac{1}{t_{j}^{2}}\right)\right] v_{r}\right) \\
& =v_{n}
\end{aligned}
$$

This means that $\hat{T}^{q} u=v$. Since $v \in \ell_{p}$, we have $\hat{T}^{q} u \in \ell_{p}$. Thus, we conclude that $u \in \ell_{p}\left(\hat{T}^{q}\right)$ for any $v \in \ell_{p}$. Hence $\hat{T}^{q}$ is surjective.

Since $\|u\|_{\ell_{p}\left(\hat{T}^{q}\right)}=\left\|\hat{T}^{q} u\right\|_{\ell_{p}}$ for any $u \in \ell_{p}\left(\hat{T}^{q}\right)$, we have

$$
\|v\|_{\ell_{p}}=\left\|\hat{T}^{q} u\right\|_{\ell_{p}}=\|u\|_{\ell_{p}\left(\hat{T}^{q}\right)}
$$

which shows that $\hat{T}^{q}$ preserves the norm, where $1 \leq p \leq \infty$. Hence, $\hat{T}^{q}$ is an isometry. As a result, the space $\ell_{p}\left(\hat{T}^{q}\right)$ is isometrically isomorphic to $\ell_{p}$ for $1 \leq p \leq \infty$.

It is known that the space $\ell_{p}$ is not a Hilbert space with $p \neq 2$. The similar result is valid for the space $\ell_{p}\left(\hat{T}^{q}\right)$ and the following theorem gives this result.

Theorem 2.3. The space $\ell_{p}\left(\hat{T}^{q}\right)$ is not an inner product space in the case $p \neq 2$. Hence, $\ell_{p}\left(\hat{T}^{q}\right)$ is not a Hilbert space for $1 \leq p<\infty$ and $p \neq 2$.

Proof. We must show that the space $\ell_{2}\left(\hat{T}^{q}\right)$ is a Hilbert space while $\ell_{p}\left(\hat{T}^{q}\right)$ is not a Hilbert space for $p \neq 2$. By Theorem 2.1, we know that $\ell_{2}\left(\hat{T}^{q}\right)$ is a Banach space with the norm $\|u\|_{\ell_{2}\left(\hat{T}^{q}\right)}=\left\|\hat{T}^{q} u\right\|_{\ell_{2}}$ and its norm can be obtained as follows:

$$
\|u\|_{\ell_{2}\left(\hat{T}^{q}\right)}=\langle u, u\rangle_{\ell_{2}\left(\hat{T}^{q}\right)}^{1 / 2}=\left\langle\hat{T}^{q} u, \hat{T}^{q} u\right\rangle_{\ell_{2}}^{1 / 2}=\left\|\hat{T}^{q} u\right\|_{\ell_{2}}
$$

for every $u \in \ell_{2}\left(\hat{T}^{q}\right)$. Hence $\ell_{2}\left(\hat{T}^{q}\right)$ is a Hilbert space.

Consider the sequences

$$
s=\left(s_{n}\right)=\left\{\begin{array}{lrl}
\frac{1}{t_{0}} & , & n=0 \\
\frac{1}{t_{0} t_{1}^{2}}+\frac{1}{t_{1}} & , & n=1 \\
t_{0} \prod_{i=0}^{n} \frac{1}{t_{i}^{2}}+t_{1} \prod_{i=1}^{n} \frac{1}{t_{i}^{2}}-\frac{Q_{1} t_{2}}{q_{2}} \prod_{i=2}^{n} \frac{1}{t_{i}^{2}} & , & n \geq 2(n \in \mathbb{N})
\end{array}\right.
$$

and

$$
t=\left(t_{n}\right)= \begin{cases}\frac{1}{t_{0}} & , \quad n=0 \\ \frac{1}{t_{0} t_{1}^{2}}-\frac{\left(Q_{0}+Q_{1}\right)}{q_{1} t_{1}} & , \quad n=1 \\ t_{0} \prod_{i=0}^{n} \frac{1}{t_{i}^{2}}-\frac{\left(Q_{0}+Q_{1}\right)}{q_{1}} t_{1} \prod_{i=1}^{n} \frac{1}{t_{i}^{2}}+\frac{Q_{1} t_{2}}{q_{2}} \prod_{i=2}^{n} \frac{1}{t_{i}^{2}} & , \quad n \geq 2(n \in \mathbb{N})\end{cases}
$$

With the $\hat{T}^{q}$-transforms of $s$ and $t$, we have the following sequences

$$
\hat{T}^{q} s=(1,1,0,0, \ldots) \text { and } \hat{T}^{q} t=(1,-1,0,0, \ldots) .
$$

Also, it can be easily seen that

$$
\|s+t\|_{\ell_{p}\left(\hat{T}^{q}\right)}^{2}+\|s-t\|_{\ell_{p}\left(\hat{T}^{q}\right)}^{2}=8 \neq 4\left(2^{2 / p}\right)=2\left(\|s\|_{\ell_{p}\left(\hat{T}^{q}\right)}^{2}+\|t\|_{\ell_{p}\left(\hat{T}^{q}\right)}^{2}\right)
$$

for $p \neq 2$. This means that the parallelogram equality cannot be satisfied by the norm of the space $\ell_{p}\left(\hat{T}^{q}\right)$ for $p \neq 2$. Therefore, this norm cannot be gained from an inner product. Therefore, the space $\ell_{p}\left(\hat{T}^{q}\right)$ with $p \neq 2$ is a Banach space but it is not a Hilbert space, where $1 \leq p<\infty$. The proof is completed.

Remark 2.2. Obviously, the space $\ell_{\infty}\left(\hat{T}^{q}\right)$ is also a Banach space but it is not a Hilbert space. 
Now, we give some theorems on inclusion relations associated with the space $\ell_{p}\left(\hat{T}^{q}\right)$.

Theorem 2.4. For $1 \leq p<q<\infty$ the inclusion relation $\ell_{p}\left(\hat{T}^{q}\right) \subset \ell_{q}\left(\hat{T}^{q}\right)$ strictly holds.

Proof. Let $1 \leq p<q<\infty$. If $u$ is any sequence in $\ell_{p}\left(\hat{T}^{q}\right)$, then its $\hat{T}^{q}$-transform $\hat{T}^{q} u$ is in $\ell_{p}$. Since the inclusion $\ell_{p} \subset \ell_{q}$ holds, $\hat{T}^{q} u$ is also in $\ell_{q}$. Hence $u \in \ell_{q}\left(\hat{T}^{q}\right)$ which means that $\ell_{p}\left(\hat{T}^{q}\right) \subset \ell_{q}\left(\hat{T}^{q}\right)$. Now, we must prove that the inclusion holds strictly. For this, there should be a sequence $\hat{v}=\left(\hat{v}_{n}\right) \in \ell_{q}$ but not in $\ell_{p}$, i.e., $\hat{v} \in \ell_{q} \backslash \ell_{p}$. The existence of $\hat{v} \in \ell_{q} \backslash \ell_{p}$ is clear since, as a well known fact, $\ell_{p} \subset \ell_{q}$ is a strict inclusion. Let define the sequence $v=\left(v_{n}\right)$ in terms of the sequence $\hat{v}$ as follows:

$$
v_{n}=\sum_{k=0}^{n} Q_{k}\left[\frac{1}{q_{k}}\left(t_{k} \prod_{j=k}^{n} \frac{1}{t_{j}^{2}}\right)-\frac{1}{q_{k+1}}\left(t_{k+1} \prod_{j=k+1}^{n} \frac{1}{t_{j}^{2}}\right)\right] \hat{v}_{k} \quad(n \in \mathbb{N}) .
$$

Then, it is clear that

$$
\hat{T}_{n}^{q}(v)=\hat{v}_{n}
$$

for every $n \in \mathbb{N}$. This shows that $\hat{T}^{q} v=\hat{v}$ and since $\hat{v} \in \ell_{q} \backslash \ell_{p}$, we have $\hat{T}^{q} v \in \ell_{q} \backslash \ell_{p}$. Hence, the sequence $v$ must be in $\ell_{q}\left(\hat{T}^{q}\right)$ but cannot be in $\ell_{p}\left(\hat{T}^{q}\right)$, that is, the inclusion $\ell_{p}\left(\hat{T}^{q}\right) \subset \ell_{q}\left(\hat{T}^{q}\right)$ is strict. The proof is completed.

Theorem 2.5. For $1 \leq p<\infty$ the inclusion $\ell_{p}\left(\hat{T}^{q}\right) \subset \ell_{\infty}\left(\hat{T}^{q}\right)$ is strict.

Proof. If $u \in \ell_{p}\left(\hat{T}^{q}\right)$, then $\hat{T}^{q} u \in \ell_{p}$. Since $\ell_{p} \subset \ell_{\infty}, \hat{T}^{q} u \in \ell_{\infty}$. Hence, $u \in \ell_{\infty}\left(\hat{T}^{q}\right)$ which shows that $\ell_{p}\left(\hat{T}^{q}\right) \subset \ell_{\infty}\left(\hat{T}^{q}\right)$. To show that this inclusion is strict, we define the sequence $v=\left(v_{n}\right)$ by

$$
v_{n}=t_{0} \prod_{i=0}^{n} \frac{1}{t_{i}^{2}}+\sum_{i=2}^{n}(-1)^{i-1} \frac{\left(Q_{i-2}+Q_{i-1}\right)}{q_{i-1}}\left(t_{i-1} \prod_{k=i-1}^{n} \frac{1}{t_{k}^{2}}\right)+(-1)^{n} \frac{\left(Q_{n-1}+Q_{n}\right)}{q_{n} t_{n}} \quad(n \in \mathbb{N}) .
$$

Then, we have for every $n \in \mathbb{N}$ that

$$
\begin{aligned}
\hat{T}_{n}^{q}(v) & =\frac{1}{Q_{n}} \sum_{k=0}^{n} q_{k}\left(t_{k} v_{k}-\frac{v_{k-1}}{t_{k}}\right) \\
& =(-1)^{n} .
\end{aligned}
$$

Then, $\hat{T}^{q} v \in \ell_{\infty} \backslash \ell_{p}$ since $\left((-1)^{n}\right) \in \ell_{\infty}$ but not in $\ell_{p}$. Thus, $v$ is in $\ell_{\infty}\left(\hat{T}^{q}\right)$ but not in $\ell_{p}\left(\hat{T}^{q}\right)$ which means that the inclusion $\ell_{p}\left(\hat{T}^{q}\right) \subset \ell_{\infty}\left(\hat{T}^{q}\right)$ strictly holds. The proof is completed.

\section{The $\alpha$-, $\beta$ - and $\gamma$-duals of the space $\ell_{p}\left(\hat{T}^{q}\right)$}

In this section, we determine the $\alpha$-, $\beta$ - and $\gamma$-duals of the sequence space $\ell_{p}\left(\hat{T}^{q}\right)$, where $1 \leq p \leq \infty$. Also, we give a sequence of the points of the space $\ell_{p}\left(\hat{T}^{q}\right)$ which forms a basis for this space.

The following known results in [38] and [39] are fundamental for our investigation.

$$
\begin{gathered}
\sup _{n} \sum_{k}\left|b_{n k}\right|^{q}<\infty . \\
\lim _{n \rightarrow \infty} b_{n k} \text { exists for all } k \in \mathbb{N} . \\
\lim _{n \rightarrow \infty} b_{n k}=0 \text { for all } k \in \mathbb{N} . \\
\sup _{K \in \mathcal{F}} \sum_{k}\left|\sum_{n \in K} b_{n k}\right|^{q}<\infty . \\
\sup _{n, k}\left|b_{n k}\right|<\infty \\
\sup _{k} \sum_{n}\left|b_{n k}\right|<\infty .
\end{gathered}
$$




$$
\begin{gathered}
\lim _{n \rightarrow \infty} \sum_{k}\left|b_{n k}\right|=\sum_{k}\left|\lim _{n \rightarrow \infty} b_{n k}\right| . \\
\lim _{n \rightarrow \infty} \sum_{k}\left|b_{n k}\right|=0 .
\end{gathered}
$$

Lemma 3.1. Let $B=\left(b_{n k}\right)$ be an infinite matrix. The following statements hold:

1. $B \in\left(\ell_{p}, \ell_{\infty}\right) \Leftrightarrow(3.1)$.

2. $B \in\left(\ell_{1}, \ell_{\infty}\right) \Leftrightarrow(3.5)$.

3. $B \in\left(\ell_{\infty}, \ell_{\infty}\right) \Leftrightarrow$ (3.1) with $q=1$.

4. $B \in\left(\ell_{p}, c\right) \Leftrightarrow$ (3.1) and (3.2).

5. $B \in\left(\ell_{1}, c\right) \Leftrightarrow$ (3.2) and (3.5).

6. $B \in\left(\ell_{\infty}, c\right) \Leftrightarrow$ (3.2) and (3.7).

7. $B \in\left(\ell_{p}, c_{0}\right) \Leftrightarrow$ (3.1) and (3.3).

8. $B \in\left(\ell_{1}, c_{0}\right) \Leftrightarrow$ (3.3) and (3.5).

9. $B \in\left(\ell_{\infty}, c_{0}\right) \Leftrightarrow$ (3.3) and (3.8).

10. $B \in\left(\ell_{p}, \ell_{1}\right) \Leftrightarrow(3.4)$.

11. $B \in\left(\ell_{1}, \ell_{1}\right) \Leftrightarrow(3.6)$.

12. $B \in\left(\ell_{\infty}, \ell_{1}\right) \Leftrightarrow(3.4)$ with $q=1$.

Now, let give two lemmas which are needed to determine the $\alpha-, \beta$ - and $\gamma$-duals of the space $\ell_{p}\left(\hat{T}^{q}\right)$, where $1 \leq p \leq \infty$.

Lemma 3.2. Let $a=\left(a_{n}\right) \in \omega$ and the matrix $\hat{B}=\left(\hat{b}_{n k}\right)$ be defined by $\hat{B}_{n}=a_{n}\left(\hat{T}_{n}^{q}\right)^{-1}$, that is,

$$
\hat{b}_{n k}=\left\{\begin{array}{cl}
0 & , \quad k>n \\
a_{n}\left(\hat{t}^{q}\right)_{n k}^{-1} & , \quad 0 \leq k \leq n
\end{array}\right.
$$

for all $k, n \in \mathbb{N}$. Then, $a \in\left(\ell_{p}\left(\hat{T}^{q}\right)\right)^{\alpha}$ if and only if $\hat{B} \in\left(\ell_{p}, \ell_{1}\right)$, where $1 \leq p \leq \infty$.

Proof. Let $\hat{u}$ be the $\hat{T}^{q}$-transform of a sequence $u=\left(u_{n}\right) \in \omega$. Then, we have

$$
a_{n} u_{n}=a_{n}\left(\hat{T}^{q}\right)_{n}^{-1}(\hat{u})=\hat{B}_{n}(\hat{u})
$$

for all $n \in \mathbb{N}$. So, from this equality it can be easily seen that $a u=\left(a_{n} u_{n}\right) \in \ell_{1}$ with $u \in \ell_{p}\left(\hat{T}^{q}\right)$ if and only if $\hat{B} \hat{u} \in \ell_{1}$ with $\hat{u} \in \ell_{p}$. This implies that $a \in\left(\ell_{p}\left(\hat{T}^{q}\right)\right)^{\alpha}$ if and only if $\hat{B} \in\left(\ell_{p}, \ell_{1}\right)$. The proof is completed.

Lemma 3.3. [40, Theorem 3.1] Let $C=\left(c_{n k}\right)$ be defined via a sequence $a=\left(a_{k}\right) \in \omega$ and the inverse matrix $V=\left(v_{n k}\right)$ of the triangle matrix $U=\left(u_{n k}\right)$ by

$$
c_{n k}=\left\{\begin{array}{cl}
0 & , \quad k>n \\
\sum_{j=k}^{n} a_{j} v_{j k} & , \quad 0 \leq k \leq n
\end{array}\right.
$$

for all $k, n \in \mathbb{N}$. Then,

$$
\begin{gathered}
\left(\ell_{p}(U)\right)^{\gamma}=\left\{a=\left(a_{k}\right) \in \omega: C \in\left(\ell_{p}, \ell_{\infty}\right)\right\} \\
\left(\ell_{p}(U)\right)^{\beta}=\left\{a=\left(a_{k}\right) \in \omega: C \in\left(\ell_{p}, c\right)\right\},
\end{gathered}
$$

where $1 \leq p \leq \infty$.

Combining Lemmas 3.1-3.3 we have;

Corollary 3.1. Let the sets $\hat{d}_{1}, \hat{d}_{2}, \hat{d}_{3}, \hat{d}_{4}, \hat{d}_{5}$ and $\hat{d}_{6}$ be defined as follows:

$$
\begin{aligned}
& \hat{d}_{1}=\left\{a=\left(a_{k}\right) \in \omega: \sup _{K \in \mathcal{F}} \sum_{k}\left|\sum_{n \in K}\left(Q_{k}\left[\frac{1}{q_{k}}\left(t_{k} \prod_{j=k}^{n} \frac{1}{t_{j}^{2}}\right)-\frac{1}{q_{k+1}}\left(t_{k+1} \prod_{j=k+1}^{n} \frac{1}{t_{j}^{2}}\right)\right]\right) a_{n}\right|^{q}<\infty\right\}, \\
& \hat{d}_{2}=\left\{a=\left(a_{k}\right) \in \omega: \sum_{j=k}^{\infty}\left(Q_{k}\left[\frac{1}{q_{k}}\left(t_{k} \prod_{i=k}^{j} \frac{1}{t_{i}^{2}}\right)-\frac{1}{q_{k+1}}\left(t_{k+1} \prod_{i=k+1}^{j} \frac{1}{t_{i}^{2}}\right)\right]\right) a_{j} \text { exists for each } k \in \mathbb{N}\right\},
\end{aligned}
$$




$$
\begin{gathered}
\hat{d}_{3}=\left\{a=\left(a_{k}\right) \in \omega: \sup _{n} \sum_{k=0}^{n}\left|\sum_{j=k}^{n}\left(Q_{k}\left[\frac{1}{q_{k}}\left(t_{k} \prod_{i=k}^{j} \frac{1}{t_{i}^{2}}\right)-\frac{1}{q_{k+1}}\left(t_{k+1} \prod_{i=k+1}^{j} \frac{1}{t_{i}^{2}}\right)\right]\right) a_{j}\right|^{q}<\infty\right\}, \\
\hat{d}_{4}=\left\{a=\left(a_{k}\right) \in \omega: \lim _{n \rightarrow \infty} \sum_{k=0}^{n}\left|\sum_{j=k}^{n}\left(Q_{k}\left[\frac{1}{q_{k}}\left(t_{k} \prod_{i=k}^{j} \frac{1}{t_{i}^{2}}\right)-\frac{1}{q_{k+1}}\left(t_{k+1} \prod_{i=k+1}^{j} \frac{1}{t_{i}^{2}}\right)\right]\right) a_{j}\right|\right\}, \\
\hat{d}_{5}=\left\{a=\left(a_{k}\right) \in \omega: \sup _{k} \sum_{n}\left|\left(Q_{k}\left[\frac{1}{q_{k}}\left(t_{k} \prod_{j=k}^{n} \frac{1}{t_{j}^{2}}\right)-\frac{1}{q_{k+1}}\left(t_{k+1} \prod_{j=k+1}^{n} \frac{1}{t_{j}^{2}}\right)\right]\right) a_{n}\right|<\infty\right\}
\end{gathered}
$$

and

$$
\hat{d}_{6}=\left\{a=\left(a_{k}\right) \in \omega: \sup _{n, k}\left|\sum_{j=k}^{n}\left(Q_{k}\left[\frac{1}{q_{k}}\left(t_{k} \prod_{i=k}^{j} \frac{1}{t_{i}^{2}}\right)-\frac{1}{q_{k+1}}\left(t_{k+1} \prod_{i=k+1}^{j} \frac{1}{t_{i}^{2}}\right)\right]\right) a_{j}\right|<\infty\right\} .
$$

Then, the following statements hold:

(a) $\left(\ell_{p}\left(\hat{T}^{q}\right)\right)^{\alpha}=\hat{d}_{1}$ and $\left(\ell_{1}\left(\hat{T}^{q}\right)\right)^{\alpha}=\hat{d}_{5}$, where $1<p \leq \infty$.

(b) $\left(\ell_{p}\left(\hat{T}^{q}\right)\right)^{\beta}=\hat{d}_{2} \cap \hat{d}_{3},\left(\ell_{\infty}\left(\hat{T}^{q}\right)\right)^{\beta}=\hat{d}_{2} \cap \hat{d}_{4}$ and $\left(\ell_{1}\left(\hat{T}^{q}\right)\right)^{\beta}=\hat{d}_{2} \cap \hat{d}_{6}$, where $1<p<\infty$.

(c) $\left(\ell_{p}\left(\hat{T}^{q}\right)\right)^{\gamma}=\hat{d}_{3}$ and $\left(\ell_{1}\left(\hat{T}^{q}\right)\right)^{\gamma}=\hat{d}_{6}$, where $1<p \leq \infty$.

Now, we give the Schauder basis of the space $\ell_{p}\left(\hat{T}^{q}\right)(1 \leq p<\infty)$.

Theorem 3.1. Let $1 \leq p<\infty$ and define the sequence $c^{(k)} \in \ell_{p}\left(\hat{T}^{q}\right)$ for every fixed $k \in \mathbb{N}$ by

$$
\left(c^{(k)}\right)_{n}=\left\{\left(Q_{k}\left[\frac{1}{q_{k}}\left(t_{k} \prod_{j=k}^{n} \frac{1}{t_{j}^{2}}\right)-\frac{1}{q_{k+1}}\left(t_{k+1} \prod_{j=k+1}^{n} \frac{1}{t_{j}^{2}}\right)\right]\right), \quad n \geq k \quad(n \in \mathbb{N}) .\right.
$$

Then the sequence $\left(c^{(k)}\right)$ is a basis for the space $\ell_{p}\left(\hat{T}^{q}\right)$, and every $u \in \ell_{p}\left(\hat{T}^{q}\right)$ has a unique representation of the form

$$
u=\sum_{k} \hat{T}_{k}^{q}(u) c^{(k)} .
$$

Proof. Let $1 \leq p<\infty$. By (3.9), it is clear that $\hat{T}^{q}\left(c^{(k)}\right)=e^{(k)} \in \ell_{p}$ and $c^{(k)} \in \ell_{p}\left(\hat{T}^{q}\right)$ for all $k \in \mathbb{N}$.

Also, let $u \in \ell_{p}\left(\hat{T}^{q}\right)$ given. For every non-negative integer $m$, we put

$$
u^{(m)}=\sum_{k=0}^{m} \hat{T}_{k}^{q}(u) c^{(k)}
$$

Then, we obtain

$$
\hat{T}^{q}\left(u^{(m)}\right)=\sum_{k=0}^{m} \hat{T}_{k}^{q}(u) \hat{T}^{q}\left(c^{(k)}\right)=\sum_{k=0}^{m} \hat{T}_{k}^{q}(u) e^{(k)}
$$

and so

$$
\hat{T}_{n}^{q}\left(u-u^{(m)}\right)=\left\{\begin{array}{cl}
0 & (0 \leq n \leq m) \\
\hat{T}_{n}^{q}(u) & (n>m)
\end{array}\right.
$$

Let $\epsilon>0$ be given. Then, there exists a non-negative integer $m_{0}$ which satisfies

$$
\sum_{n=m_{0}+1}^{\infty}\left|\hat{T}_{n}^{q}(u)\right|^{p} \leq\left(\frac{\epsilon}{2}\right)^{p}
$$

So, we obtain for every $m \geq m_{0}$ that

$$
\left\|u-u^{(m)}\right\|_{\ell_{p}\left(\hat{T}^{q}\right)}=\left(\sum_{n=m+1}^{\infty}\left|\hat{T}_{n}^{q}(u)\right|^{p}\right)^{1 / p} \leq\left(\sum_{n=m_{0}+1}^{\infty}\left|\hat{T}_{n}^{q}(u)\right|^{p}\right)^{1 / p} \leq \frac{\epsilon}{2}<\epsilon
$$


which indicates that $\lim _{m \rightarrow \infty}\left\|u-u^{(m)}\right\|_{\ell_{p}\left(\hat{T}^{q}\right)}=0$ and hence $u$ is shown as in (3.10).

Finally, we must prove that the representation (3.10) of $u \in \ell_{p}\left(\hat{T}^{q}\right)$ is unique. Assume that $u=\sum_{k} \mu_{k}(u) c^{(k)}$. The continuity of the linear transformation $\hat{T}^{q}: \ell_{p}\left(\hat{T}^{q}\right) \rightarrow \ell_{p}$ which is defined in the proof of Theorem 2.2 is clear, we have

$$
\hat{T}_{n}^{q}(u)=\sum_{k} \mu_{k}(u) \hat{T}_{n}^{q}\left(c^{(k)}\right)=\sum_{k} \mu_{k}(u) \delta_{n k}=\mu_{n}(u) \quad(n \in \mathbb{N}) .
$$

Hence, the representation (3.10) of $u \in \ell_{p}\left(\hat{T}^{q}\right)$ is unique. The proof is completed.

\section{Characterization of some matrix transformations on $\ell_{p}\left(\hat{T}^{q}\right)$}

In this section of the study, we obtain the characterization of the classes $\left(\ell_{p}\left(\hat{T}^{q}\right), \lambda\right),\left(\lambda, \ell_{p}\left(\hat{T}^{q}\right)\right)$, where $1 \leq p \leq \infty$, $\lambda \in\left\{\ell_{1}, c_{0}, c, \ell_{\infty}\right\}$ and $\mu \in\left\{\ell_{1}, \ell_{\infty}\right\}$.

Throughout this section, we write $b(n, k)=\sum_{j=0}^{n} b_{j k}$ for given an infinite matrix $B=\left(b_{n k}\right)$, where $n, k \in \mathbb{N}$.

Firstly, we give a theorem which is essential for our results.

Theorem 4.1. Let $1 \leq p \leq \infty$. Then, we have $B=\left(b_{n k}\right) \in\left(\ell_{p}\left(\hat{T}^{q}\right), \lambda\right)$ if and only if

$$
\begin{gathered}
E^{(m)}=\left(e_{n k}^{(m)}\right) \in\left(\ell_{p}, c\right) \text { for all } n \in \mathbb{N}, \\
E=\left(e_{n k}\right) \in\left(\ell_{p}, \lambda\right),
\end{gathered}
$$

where $e_{n k}^{(m)}=\left\{\begin{array}{cl}0 & , \quad k>m \\ \sum_{j=k}^{m} Q_{k}\left[\frac{1}{q_{k}}\left(t_{k} \prod_{i=k}^{j} \frac{1}{t_{i}^{2}}\right)-\frac{1}{q_{k+1}}\left(t_{k+1} \prod_{i=k+1}^{j} \frac{1}{t_{i}^{2}}\right)\right] b_{n j}, \quad 0 \leq k \leq m\end{array}\right.$

and $e_{n k}=\sum_{j=k}^{\infty} Q_{k}\left[\frac{1}{q_{k}}\left(t_{k} \prod_{i=k}^{j} \frac{1}{t_{i}^{2}}\right)-\frac{1}{q_{k+1}}\left(t_{k+1} \prod_{i=k+1}^{j} \frac{1}{t_{i}^{2}}\right)\right] b_{n j}$ for all $k, m, n \in \mathbb{N}$.

Proof. For the proof, we follow the similar tecnique due to Kirişçi and Başar [4]. Let $B=\left(b_{n k}\right) \in\left(\ell_{p}(T), \lambda\right)$ and $u=\left(u_{k}\right) \in \ell_{p}\left(\hat{T}^{q}\right)$. By (2.2), we have

$$
\begin{aligned}
\sum_{k=0}^{m} b_{n k} u_{k} & =\sum_{k=0}^{m} b_{n k}\left(\sum_{j=0}^{k} Q_{j}\left[\frac{1}{q_{j}}\left(t_{j} \prod_{i=j}^{k} \frac{1}{t_{i}^{2}}\right)-\frac{1}{q_{j+1}}\left(t_{j+1} \prod_{i=j+1}^{j} \frac{1}{t_{i}^{2}}\right)\right]\right) \hat{u}_{j} \\
& =\sum_{k=0}^{m}\left(\sum_{j=k}^{m} Q_{k}\left[\frac{1}{q_{k}}\left(t_{k} \prod_{i=k}^{j} \frac{1}{t_{i}^{2}}\right)-\frac{1}{q_{k+1}}\left(t_{k+1} \prod_{i=k+1}^{j} \frac{1}{t_{i}^{2}}\right)\right] b_{n j}\right) \hat{u}_{k} \\
& =\sum_{k=0}^{m} e_{n k}^{(m)} \hat{u}_{k} \\
& =E_{n}^{(m)}(\hat{u})
\end{aligned}
$$

for all $m, n \in \mathbb{N}$. Since $B u$ exists, $E^{(m)}$ belongs to the class $\left(\ell_{p}, c\right)$. Letting $m \rightarrow \infty$ in the last equality, we obtain $B u=U \hat{u}$ which gives the result $E \in\left(\ell_{p}, \lambda\right)$.

Conversely, suppose the conditions (4.1), (4.2) hold and take any $u \in \ell_{p}(T)$. Then, we have $\left(e_{n k}\right)_{k \in \mathbb{N}} \in \ell_{p}^{\beta}$ which gives together with (4.1) that $B_{n}=\left(b_{n k}\right)_{k \in \mathbb{N}} \in\left(\ell_{p}\left(\hat{T}^{q}\right)\right)^{\beta}$ for all $n \in \mathbb{N}$. Thus, $B u$ exists. Therefore, we derive by the above equality as $m \rightarrow \infty$ that $B u=E \hat{u}$, and this shows that $B \in\left(\ell_{p}\left(\hat{T}^{q}\right), \lambda\right)$.

The following conditions are necessary for our study:

$$
\begin{gathered}
\sup _{n} \sum_{k}\left|e_{n k}\right|^{q}<\infty . \\
\lim _{n \rightarrow \infty} e_{n k} \text { exists for all } k \in \mathbb{N} . \\
\lim _{n \rightarrow \infty} e_{n k}=0 \text { for all } k \in \mathbb{N} .
\end{gathered}
$$




$$
\begin{gathered}
\sup _{K \in \mathcal{F}} \sum_{k}\left|\sum_{n \in K} e_{n k}\right|^{q}<\infty . \\
\sup _{n, k}\left|e_{n k}\right|<\infty . \\
\sup _{k} \sum_{n}\left|e_{n k}\right|<\infty . \\
\lim _{n \rightarrow \infty} \sum_{k}\left|e_{n k}\right|=\sum_{k}\left|\lim _{n \rightarrow \infty} e_{n k}\right| . \\
\lim _{n \rightarrow \infty} \sum_{k}\left|e_{n k}\right|=0 . \\
\lim _{m \rightarrow \infty} e_{n k}^{(m)} \operatorname{exists}(\forall n, k \in \mathbb{N}), \\
\sup _{m, k}\left|e_{n k}^{(m)}\right|<\infty(\forall n \in \mathbb{N}) \\
\sup _{m} \sum_{k=0}^{m}\left|e_{n k}^{(m)}\right|^{q}<\infty \\
\lim _{m \rightarrow \infty} \sum_{k=0}^{m}\left|e_{n k}^{(m)}\right|=\sum_{k}\left|e_{n k}\right| \text { for each } n \in \mathbb{N} \\
\sup _{N, K \in \mathcal{F}}\left|\sum_{n \in N} \sum_{k \in K} e_{n k}\right|<\infty
\end{gathered}
$$

We obtain the following results by combining Theorem 4.1 and previous conditions.

Theorem 4.2. The following statements hold:

1. $B=\left(b_{n k}\right) \in\left(\ell_{1}\left(\hat{T}^{q}\right), \ell_{\infty}\right) \Leftrightarrow(4.7),(4.11)$ and (4.12).

2. $B=\left(b_{n k}\right) \in\left(\ell_{p}\left(\hat{T}^{q}\right), \ell_{\infty}\right) \Leftrightarrow(4.3)$, (4.11) and (4.13).

3. $B=\left(b_{n k}\right) \in\left(\ell_{\infty}\left(\hat{T}^{q}\right), \ell_{\infty}\right) \Leftrightarrow(4.11),(4.14)$ and (4.3) with $q=1$.

4. $B=\left(b_{n k}\right) \in\left(\ell_{1}\left(\hat{T}^{q}\right), c\right) \Leftrightarrow(4.4),(4.7),(4.11)$ and (4.12).

5. $B=\left(b_{n k}\right) \in\left(\ell_{p}\left(\hat{T}^{q}\right), c\right) \Leftrightarrow(4.3),(4.4),(4.11)$ and (4.13).

6. $B=\left(b_{n k}\right) \in\left(\ell_{\infty}\left(\hat{T}^{q}\right), c\right) \Leftrightarrow(4.4),(4.9),(4.11)$ and (4.14).

7. $B=\left(b_{n k}\right) \in\left(\ell_{1}\left(\hat{T}^{q}\right), c_{0}\right) \Leftrightarrow(4.5),(4.7),(4.11)$ and (4.12).

8. $B=\left(b_{n k}\right) \in\left(\ell_{p}\left(\hat{T}^{q}\right), c_{0}\right) \Leftrightarrow(4.3),(4.5),(4.11)$ and (4.13).

9. $B=\left(b_{n k}\right) \in\left(\ell_{\infty}\left(\hat{T}^{q}\right), c_{0}\right) \Leftrightarrow$ (4.5), (4.10), (4.11) and (4.14).

10. $B=\left(b_{n k}\right) \in\left(\ell_{1}\left(\hat{T}^{q}\right), \ell_{1}\right) \Leftrightarrow(4.8),(4.11)$ and (4.12).

11. $B=\left(b_{n k}\right) \in\left(\ell_{p}\left(\hat{T}^{q}\right), \ell_{1}\right) \Leftrightarrow(4.6),(4.11)$ and $(4.13)$.

12. $B=\left(b_{n k}\right) \in\left(\ell_{\infty}\left(\hat{T}^{q}\right), \ell_{1}\right) \Leftrightarrow(4.7),(4.11)$ and $(4.14)$.

By using Theorem 4.2, we derive the following result:

Corollary 4.1. The following statements hold:

1. $B=\left(b_{n k}\right) \in\left(\ell_{1}\left(\hat{T}^{q}\right), c s_{0}\right) \Leftrightarrow(4.5)$, (4.7) and (4.11), (4.12).

2. $B=\left(b_{n k}\right) \in\left(\ell_{p}\left(\hat{T}^{q}\right), c s_{0}\right) \Leftrightarrow(4.3),(4.5)$ and (4.11), (4.13).

3. $B=\left(b_{n k}\right) \in\left(\ell_{\infty}\left(\hat{T}^{q}\right), c s_{0}\right) \Leftrightarrow(4.5),(4.10)$ and (4.11), (4.14).

4. $B=\left(b_{n k}\right) \in\left(\ell_{1}\left(\hat{T}^{q}\right), c s\right) \Leftrightarrow(4.4),(4.7)$ and $(4.11),(4.12)$.

5. $B=\left(b_{n k}\right) \in\left(\ell_{p}\left(\hat{T}^{q}\right), c s\right) \Leftrightarrow(4.3),(4.4)$ and (4.11), (4.13).

6. $B=\left(b_{n k}\right) \in\left(\ell_{\infty}\left(\hat{T}^{q}\right), c s\right) \Leftrightarrow(4.4),(4.9)$ and (4.11), (4.14).

7. $B=\left(b_{n k}\right) \in\left(\ell_{1}\left(\hat{T}^{q}\right), b s\right) \Leftrightarrow(4.7)$ and (4.11), (4.12).

8. $B=\left(b_{n k}\right) \in\left(\ell_{p}\left(\hat{T}^{q}\right), b s\right) \Leftrightarrow(4.3)$ and $(4.11),(4.13)$.

9. $B=\left(b_{n k}\right) \in\left(\ell_{\infty}\left(\hat{T}^{q}\right), b s\right) \Leftrightarrow$ (4.3) with $q=1$ and (4.11), (4.14)

hold with $d(n, k)$ instead of $d_{n k}$. 
Now, we introduce the matrix transformations from the space $\lambda \in\left\{\ell_{1}, c_{0}, c, \ell_{\infty}\right\}$ to $\ell_{p}\left(\hat{T}^{q}\right)$, where $1 \leq p \leq \infty$. Before this, we give the necessary and sufficient conditions for the matrix transformation $B$ is in $\left(\lambda, \ell_{p}\right)$.

Lemma 4.1. The following statements hold:

(a) $B \in\left(\ell_{\infty}, \ell_{p}\right)=\left(c, \ell_{p}\right)=\left(c_{0}, \ell_{p}\right)$ if and only if

$$
\sup _{K \in \mathcal{F}} \sum_{k}\left|\sum_{n \in K} b_{n k}\right|^{p}<\infty, \text { where } 1 \leq p<\infty .
$$

(b) $B \in\left(\ell_{\infty}, \ell_{\infty}\right)=\left(c, \ell_{\infty}\right)=\left(c_{0}, \ell_{\infty}\right)$ if and only if

$$
\sup _{n} \sum_{k}\left|b_{n k}\right|<\infty
$$

(c) $B \in\left(\ell_{1}, \ell_{p}\right)$ if and only if

$$
\sup _{k} \sum_{n}\left|b_{n k}\right|^{p}<\infty, \text { where } 1 \leq p<\infty
$$

When we change the roles of the spaces $\ell_{p}\left(\hat{T}^{q}\right)$ and $\ell_{p}$ with $\lambda$ in Theorem 4.1, we obtain the following theorem.

Theorem 4.3. Assume that the terms of the infinite matrices $B=\left(b_{n k}\right)$ and $\tilde{B}=\left(\tilde{b}_{n k}\right)$ satisfies the following relation

$$
\tilde{b}_{n k}=\sum_{r=0}^{n-1}\left(\frac{q_{r}}{Q_{n}} t_{r}-\frac{q_{r+1}}{Q_{n}} \frac{1}{t_{r+1}}\right) b_{r k}+\frac{q_{n}}{Q_{n}} t_{n} b_{n k}
$$

for all $k, n \in \mathbb{N}$ and $\lambda$ be any given sequence space. Then, $B \in\left(\lambda, \ell_{p}\left(\hat{T}^{q}\right)\right)$ if and only if $\tilde{B} \in\left(\lambda, \ell_{p}\right)$, where $1 \leq p \leq \infty$.

Proof. Let $u=\left(u_{k}\right) \in \lambda$. Then, by using the relation (4.19) one can easily obtain the following equality

$$
\sum_{k=0}^{m} \tilde{b}_{n k} u_{k}=\sum_{k=0}^{m}\left(\sum_{r=0}^{n-1}\left(\frac{q_{r}}{Q_{n}} t_{r}-\frac{q_{r+1}}{Q_{n}} \frac{1}{t_{r+1}}\right) b_{r k}+\frac{q_{n}}{Q_{n}} t_{n} b_{n k}\right) u_{k} \text { for all } m, n \in \mathbb{N}
$$

which yields as $m \rightarrow \infty$ that $\left(\tilde{B}_{n}(u)\right)=\left(\hat{T}_{n}^{q}(B u)\right)$. Therefore, we conclude that $B u \in \ell_{p}\left(\hat{T}^{q}\right)$ for $u \in \lambda$ if and only if $\tilde{B} u \in \ell_{p}$ for $u \in \lambda$, where $1 \leq p \leq \infty$. The proof is completed.

By combining Lemma 4.1 and Theorem 4.3 , we obtain the following results:

Corollary 4.2. Let the matrices $B=\left(b_{n k}\right)$ and $\tilde{B}=\left(\tilde{b}_{n k}\right)$ be connected by (4.19). Then, we obtain:

(a) $B=\left(b_{n k}\right) \in\left(\ell_{\infty}, \ell_{1}\left(\hat{T}^{q}\right)\right)=\left(c, \ell_{1}\left(\hat{T}^{q}\right)\right)=\left(c_{0}, \ell_{1}\left(\hat{T}^{q}\right)\right)$ if and only if (4.16) holds with $p=1$ and $\tilde{b}_{n k}$ instead of $b_{n k}$.

(b) $B=\left(b_{n k}\right) \in\left(\ell_{1}, \ell_{1}\left(\hat{T}^{q}\right)\right)$ if and only if (4.18) holds with $p=1$ and $\tilde{b}_{n k}$ instead of $b_{n k}$.

Corollary 4.3. Let the matrices $B=\left(b_{n k}\right)$ and $\tilde{B}=\left(\tilde{b}_{n k}\right)$ be connected by (4.19). For $1<p<\infty$, we obtain:

(a) $B=\left(b_{n k}\right) \in\left(\ell_{\infty}, \ell_{p}\left(\hat{T}^{q}\right)\right)=\left(c, \ell_{p}\left(\hat{T}^{q}\right)\right)=\left(c_{0}, \ell_{p}\left(\hat{T}^{q}\right)\right)$ if and only if $(4.16)$ holds with $\tilde{b}_{n k}$ instead of $b_{n k}$.

(b) $B=\left(b_{n k}\right) \in\left(\ell_{1}, \ell_{p}\left(\hat{T}^{q}\right)\right)$ if and only if (4.18) holds with $\tilde{b}_{n k}$ instead of $b_{n k}$.

Corollary 4.4. Let the matrices $B=\left(b_{n k}\right)$ and $\tilde{B}=\left(\tilde{b}_{n k}\right)$ be connected by (4.19). Then, we obtain:

(a) $B=\left(b_{n k}\right) \in\left(\ell_{\infty}, \ell_{\infty}\left(\hat{T}^{q}\right)\right)=\left(c, \ell_{\infty}\left(\hat{T}^{q}\right)\right)=\left(c_{0}, \ell_{\infty}\left(\hat{T}^{q}\right)\right)$ if and only if (4.17) holds with $\tilde{b}_{n k}$ instead of $b_{n k}$.

(b) $B=\left(b_{n k}\right) \in\left(\ell_{1}, \ell_{\infty}\left(\hat{T}^{q}\right)\right)$ if and only if (3.5) holds with $\tilde{b}_{n k}$ instead of $b_{n k}$.

\section{References}

[1] Bektaş, Ç. A., Et, M. and Çolak, R., Generalized difference sequence spaces and their dual spaces. J. Math. Anal. Appl. 292 (2004), no. 2, 423-432.

[2] Aydın, C. and Başar, F., Some new sequence spaces which include the spaces $\ell_{p}$ and $\ell_{\infty}$. Demonstratio Math. 38 (2005), no. 3, 641-656.

[3] Mursaleen, M., Başar, F. and Altay, B., On the Euler sequence spaces which include the spaces $\ell_{p}$ and $\ell_{\infty}$ II. Nonlinear Anal. 65 (2006), no. 3, 707-717. 
[4] Kirişçi, M. and Başar, F., Some new sequence spaces derived by the domain of generalized difference matrix. Comput. Math. Appl. 60 (2010), no. 5, 1299-1309.

[5] Polat, H., Karakaya, V. and Şimşek, N., Difference sequence spaces derived by using a generalized weighted mean. Appl. Math. Lett. 24 (2011), no. 5, 608-614.

[6] Mursaleen, M. and Noman, A. K., On some new sequence spaces of non-absolute type related to the spaces $\ell_{p}$ and $\ell_{\infty}$ I. Filomat 25 (2011), no. 2, 33-51.

[7] Savaş, E., Matrix transformations between some new sequence spaces. Tamkang J. Math. 19 (1988), no. 4, 75-80.

[8] Candan, M., A new sequence space isomorphic to the space $\left.\ell_{(} p\right)$ and compact operators. J. Math. Comput. Sci. 4 (2014), no. 2, 306-334.

[9] Kara, E. E. and İlkhan, M., Some properties of generalized Fibonacci sequence spaces. Linear Multilinear Algebra 64 (2016), no. 11, 2208-2223.

[10] Demiriz, S. and Duyar, O., Domain of the generalized double Cesaro matrix in some paranormed spaces of double sequences. Tbil. Math. J. 10 (2017), no. 2, 43-56.

[11] Altay, B. and Kama, R., On Cesaro summability of vector valued multiplier spaces and operator valued series. Positivity 22 (2018), no. 2, 575-586.

[12] Das, A. and Hazarika, B., Some new Fibonacci difference spaces of non-absolute type and compact operators. Linear Multilinear Algebra, 65 (2017), no. 12, 2551-2573.

[13] S. Demiriz and C. Çakan, Some topological and geometrical properties of a new difference sequence space. Abstr. Appl. Anal. 2011 (2011), Article ID 213878, 14 pages.

[14] Ellidokuzoğlu H. B. and S. Demiriz, Euler-Riesz difference sequence spaces. Turk. J. Math. Comput. Sci. 7 (2017), 63-72.

[15] Kiriş̧̧i, M., The application domain of infinite matrices with algorithms. Univers. J. Math. Appl. 1 (2018), no. 1, $1-9$.

[16] Ellidokuzoğlu, H. B., Demiriz, S. and Köseoğlu, A., On the paranormed binomial sequence spaces. Univers. J. Math. Appl. 1 (2018), no. 3, 137-147.

[17] Hazar, G. C. and Sarı̈öl, M. A., On absolute Nörlund spaces and matrix operators. Acta Math. Sin. (Engl. Ser.) 34 (2018), no. 5, 812-826.

[18] Hazar, G. C. and Sarıgöl, M. A., Absolute Cesàro series spaces and matrix operators. Acta Appl. Math. 154 (2018), no. 1, 153-165.

[19] Gökçe, F. and Sarıgöl, M. A., Generalization of the absolute Cesàro space and some matrix transformations. Numer. Funct. Anal. Optim. 40 (2019), no. 9, 1039-1052.

[20] İlkhan, M. and Kara, E. E., A new Banach space defined by Euler Totient matrix operator, Oper. Matrices 13 (2019), no. 2, 527-544.

[21] Başar, F., Summability Theory and Its Applications, Bentham Science Publishers, İstanbul, 2012.

[22] Kızmaz, H., On certain sequence spaces. Can. Math. Bull. 24 (1981), no. 2, 169-176.

[23] Et, M. and Çolak, R., On some generalized difference sequence spaces. Soochow J. Math. 21 (1995), 377-386.

[24] Altay, B. and Başar, F., The matrix domain and the fine spectrum of the difference operator $\Delta$ on the sequence space $\ell_{p}, 0<p<1$. Commun. Math. Anal. 2 (2007), no. 2, 1-11.

[25] Başar, F. and Altay, B., On the space of sequences of $p$-bounded variation and related matrix mappings. Ukr. Math. J. 55 (2003), 136-147.

[26] Çolak, R., Et, M. and Malkowsky, E., Some topics of sequence spaces, Lecture Notes in Mathematics, Firat Univ. Press, Firat Univ. Elazı ̆̆, Turkey, 1-63, 2004. 
[27] Kara, E. E., Some topological and geometrical properties of new Banach sequence spaces. J. Inequal. Appl. 2013:38 (2013), 15 pages.

[28] Altay, B., Başar, F., On the paranormed Riesz sequence spaces of non-absolute type. Southeast Asian Bull. Math. 26 (2003), no. 5, 701-715.

[29] Kara, E. E. and İlkhan, M., On some Banach sequence spaces derived by a new band matrix. British J. Math. Comput. Sci. 9 (2015), no. 2, 141-159.

[30] Sarıgöl, M. A., On difference sequence spaces. J. Karadeniz Tech. Univ., Fac. Arts Sci., Ser. Math.-Phys. 10 (1987), 63-71.

[31] Et, M., On some difference sequence spaces. Turkish J. Math. 17 (1993), 18-24.

[32] Sönmez, A, Almost convergence and triple band matrix. Math. Comput. Model. 57 (2013), 2393-2402.

[33] Mursaleen, M. and Noman, A. K., On some new difference sequence spaces of non-absolute type. Math. Comput. Model. 52 (2010), 603-617.

[34] Altay, B., Başar, F. and Mursaleen, M., On the Euler sequence spaces which include the spaces $\ell_{p}$ and $\ell_{\infty}$ I. Inform. Sci. 176 (2006), no. 10, 1450-1462.

[35] Aydın, C. and Başar, F., Some new difference sequence spaces. Appl. Math. Comput. 157 (2004), no. 3, 677-693.

[36] Candan, M., Domain of the double sequential band matrix in the classical sequence spaces. J. Inequal. Appl. 2012:281 (2012), 15 pages.

[37] Candan, M., Almost convergence and double sequential band matrix. Acta Math. Sci. 34B (2014), no. 2, 354-366.

[38] Stieglitz, M. and Tietz, H., Matrix transformationen von folgenraumen eine ergebnisübersicht. Math. Z. 154 (1977), 1-16.

[39] Maddox, I. J., Lecture Notes in Mathematics. Infinite Matrices of Operators, Springer-Verlag, Berlin Heidelberg New York, 1980.

[40] Altay, B. and Başar, F., Certain topological properties and duals of the matrix domain of a triangle matrix in a sequence space. J. Math. Anal. Appl. 336 (2007), no. 1, 632-645.

\section{Affiliations}

PinAR ZeNGin AlP

AdDRESS: Düzce University, Dept. of Mathematics, 81620, Düzce-Turkey.

E-MAIL: pinarzenginalp@gmail.com

ORCID ID:0000-0001-9699-7199

MERVE İLKHAN

AdDRESS: Düzce University, Dept. of Mathematics, 81620, Düzce-Turkey.

E-MAIL: merveilkhan@duzce.edu.tr

ORCID ID:0000-0002-0831-1474 